\title{
DEVELOPMENT OF AN ADVANCED METHOD OF FINDING SOLUTIONS FOR NEURO-FUZZY EXPERT SYSTEMS OF ANALYSIS OF THE RADIOELECTRONIC SITUATION
}

\author{
Hennadii Pievtsov \\ Deputy Head of University ${ }^{1}$ \\ info@hups.mil.gov.ua \\ Oleksandr Turinskyi \\ Head of University ${ }^{1}$ \\ red.hnups@gmail.com \\ Ruslan Zhyvotovskyi \\ Research Department of Development of Anti-Aircraft Missile Systems and Complexes ${ }^{2}$ \\ ruslan_zvivotov@ukr.net \\ Oleg Sova \\ Department of Automated Control Systems \\ Military Institute of Telecommunications and Informatization named after Heroes of Kruty \\ 45/1 Moskovsky str., Kyiv, Ukraine, 010011 \\ soy_135@ukr.net \\ Oleksii Zvieriev \\ Research Department of Development of Anti-Aircraft Missile Systems and Complexes ${ }^{2}$ \\ gans7995@gmail.com \\ Boris Lanetskii \\ Leading Researcher \\ Scientific Center ${ }^{1}$ \\ info@hups.mil.gov.ua \\ Andrii Shyshatskyi \\ Research Department of Electronic Warfare Development ${ }^{2}$ \\ ierikon13@gmail.com \\ ${ }^{1}$ Ivan Kozhedub Kharkiv National Air Force University \\ 77/79 Sumska str., Kharkiv, Ukraine, 61023 \\ ${ }^{2}$ Central Scientific Research Institute of the Army of the Armed Forces of Ukraine \\ 28 Povitroflotsky ave., Kyiv, Ukraine, 03049
}

\footnotetext{
Abstract

Nowadays, artificial intelligence has entered into all spheres of our life. The system of analysis of the electronic environment is not an exception. However, there are a number of problems in the analysis of the electronic environment, namely the signals. They are analyzed in a complex electronic environment against the background of intentional and natural interference. Also, the input signals do not match the standards due to the influence of different types of interference. Interpretation of signals depends on the experience of the operator, the completeness of additional information on a specific condition of uncertainty. The best solution in this situation is to integrate with the data of the information system analysis of the electronic environment and artificial neural networks. Their advantage is also the ability to work in real time and quick adaptation to specific situations. These circumstances cause uncertainty in the conditions of the task of signal recognition and fuzzy statements in their interpretation, when the additional involved information may be incomplete and the operator makes decisions based on their experience.

That is why, in this article, an improved method for finding solutions for neuro-fuzzy expert systems of analysis of the electronic environment is developed.
} 
Improving the efficiency of information processing (reducing the error) of evaluation is achieved through the use of neuro-fuzzy artificial neural networks that are evolving and learning not only the synaptic weights of the artificial neural network, but also the type and parameters of the membership function. High efficiency of information processing is also achieved through training in the architecture of artificial neural networks by taking into account the type of uncertainty of the information that has to be assessed and work with clear and fuzzy products. This reduces the computational complexity of decision-making and absence of accumulation of an error of training of artificial neural networks as a result of processing of the arriving information on an input of artificial neural networks. The use of the proposed method was tested on the example of assessing the state of the electronic environment. This example showed an increase in the efficiency of assessment at the level of 20-25\% on the efficiency of the processing information.

Keywords: artificial intelligence; electronic environment; intelligent systems; decision-making support systems.

DOI: $10.21303 / 2461-4262.2020 .001353$

\section{Introduction}

Nowadays, many areas of human activity use artificial intelligence approaches to solve important practical problems. Expert systems have been successfully used in complex technical systems to solve informal or poorly formalized tasks, such as training, diagnostics, forecasting, control and measurement [1-3].

This class of intelligent information systems is characterized by the fact that they are able to model the process of thinking of the expert in making a decision and explain why this or that result was obtained. This is achieved by implementing the procedure of logical inference on formalized knowledge about the subject area, about the processes that take place in it, about the laws that govern these processes $[2,4]$.

The main element of any task of analysis of the radioelectronic environment (REE) is to compare the obtained signals with the reference values that are available in the database.

However, there are a number of difficulties and problems in the analysis of REE:

1. Signals are analyzed in a complex electronic environment against the background of intentional and natural interference.

2. The input signals do not match the standards due to the influence of different types of interference.

3. Interpretation of signals depends on the experience of the operator, the completeness of additional information on a particular task (conditions of uncertainty).

The best solution in this situation is to integrate with the analysis data of the information system analysis of REE and artificial neural networks (ANN).

These circumstances lead to recourse to the theory of expert systems, where one of the important limitations in their use is the difficulty of formulating rules for machining. And when it is used in conjunction with the analysis system REE, it is difficult to formulate rules for the transfer and transformation of the assessment of the assessment of areas from the source of knowledge to the program. In this form, an effective methodology for recording, storing and using expert knowledge should be developed in the expert system for the operational sampling of knowledge [4].

An alternative method of capturing expertise without using rules is to use artificial neural networks, using their ability to generalize, self-learn and retrain. Their advantages are also the ability to work in real time and quick adaptation to specific situations.

These circumstances cause uncertainty in the conditions of the task of signal recognition and fuzzy statements in their interpretation, when the involved additional information may be incomplete and the operator makes decisions based on their experience.

To achieve this aim, the following objectives are set:

- to carry out mathematical statement of the problem of the analysis of a radio-electronic situation;

- to develop an improved method for finding solutions for neuro-fuzzy expert systems of analysis of the electronic environment;

- to evaluate the effectiveness of the proposed method. 


\section{Mathematical formulation of the problem of analysis of the electronic situation}

Let it be that a vector model is obtained as a result of the classification of signals by spectral characteristics. The model determines the shape of the studied signals, so the studied signals are divided into elementary components according to the characteristics that make up the set of $V$ elementary components of the analyzed signal.

The electronic environment, that is stored in computer memory in digital form, will be represented as a matrix $R$ of the $(M * N)$ dimension, and has the form [2, 4]:

$$
R=\left\|r_{i, j}\right\|,
$$

where

$$
i=1, \ldots, M ; j=1, \ldots, N
$$

Each elements of the matrix $R$ are vector of parameters that characterize each $(i, j)$-th elementary parameter of the signal on some $m$-th $(m=1, \ldots, T)$ set of thematic properties:

$$
r_{i, j}=\left(r_{i, j}^{1}, \ldots, r_{i, j}^{T}\right)
$$

the history of the components of the $r_{i, j}$ vectors does not play a big role.

Then, if to set a set of thematic properties $\left\{P_{n}\right\},(n=1, \ldots, K)$, according to which it is necessary to classify the studied signals and threshold values of constraints on the whole set of thematic properties, it is necessary for each $P_{n}$ of their threshold constraints to match many elementary components $P_{n} V_{t} \in V,(1 \leq t \leq(M \times N))^{n}$ of the signal $P_{n}$.

Then, showing all sorts of variants of the values of the features within the thresholds of constraints for each to the $k$-th reference table and have a matrix of estimates $\Gamma^{k l}$ at the output

$$
\Gamma^{k l}=\left\|q_{u, v}^{k l}\right\|, q_{u, v}^{k l} \in[0,1],
$$

where the answer $l$ means that the set of features and their thresholds of restrictions makes a decision about belonging to one of the classes of belonging and 0 .

However, even with all the advantages of neuro-fuzzy expert, they unfortunately have certain disadvantages. Here are the main ones [6-9]:

- accumulation of evaluation error during fuzzification and defuzzification procedures;

- architecture of the artificial neural network used to form knowledge bases has a rigid architecture, and is not able to adapt during the calculations;

- learning of an artificial neural network is limited only to learning of synaptic weights between neurons;

- the low productivity of methods of the decisions search at insignificant volume of rules;

- the great computational complexity of the methods of the solutions finding.

Therefore, it is necessary to develop a method for finding a solution for neuro-fuzzy expert systems for analyzing the electronic environment.

\section{The development of an improved method for finding solutions for neuro-fuzzy expert sys- tems for analysis of the electronic environment}

The Rete method was chosen as the basis for the development of an improved method for finding solutions for neuro-fuzzy expert systems for the analysis of the electronic environment $[5,10,11]$. The main disadvantage of the Rete method is the fact that it works only with clear products, which does not allow it to be used while processing the different types of data.

The algorithm for implementing the proposed search method is shown in Fig. 1.

Step 1. Enter the input data for the analysis of the electronic environment (step 1 in Fig. 1).

At this stage, it is possible to make the introduction of the initial electronic environment, which is typical of this region. 
Step 2. Formation of knowledge base (KB) taking into account uncertainty.

At this stage, the formation of KB on the basis of expressions (4)-(17). While converting the values of REE into fuzzy rules, the value of uncertainty about the sources of radio radiation is taken into account, according to expressions (10)-(12) [2].

The formal model of the neuro-fuzzy rule base will look like (4)

$$
\left\{P_{n}\right\}=\{\text { Rule }\}
$$

where Rule is a rule of the neuro-fuzzy expert system. Each rule is defined as follows (5)

$$
\text { Rule }=<C \rightarrow S>
$$

where $C$ is the condition of the rule, $S$ is the consequence of the rule.

Since the model must provide a representation of the grammatical structure of the rules from different types of nested conditions, a recursive mechanism will be used to describe the nodes and end vertices of the rule conditions. Parameter $C$ is defined as follows (6):

$$
\tilde{N}=<C_{l}, R, C_{r}>
$$

where $C_{l}$ are the left node rule conditions, $R$ is the relationship between rule nodes, $C_{r}$ are the right node rule conditions.

Let's consider the following parameters.

$$
\begin{gathered}
C_{l}=F C_{l}\|\operatorname{Null}\| C, \\
C_{r}=F C_{r}\|\operatorname{Null}\| C,
\end{gathered}
$$

where $F C_{l}$ is the left final triple of the rule condition, $F C_{r}$ is the right final triple of the rule condition.

Formulas (7) and (8) allow to describe conditions with different degrees of nesting.

$$
\begin{aligned}
& F C_{l}=<L, Z, W>, \\
& F C_{r}=<L, Z, W>,
\end{aligned}
$$

where $L$ is the linguistic variable, $Z$ is the condition sign

$$
Z=\{<,>,<=,>=,=, !=\}
$$

$W$ is the value of the condition, which is determined as follows (11):

$$
W=L \| V,
$$

where $L$ is the linguistic variable, $V$ is the fixed value (12).

$$
V=T_{i} \| \text { const, }
$$

where $T_{i}$ is the value of a fuzzy variable from the term sets of a linguistic variable, const is a constant. This model allows the use of not only linguistic variables but also classical variables. In this case, their values can also be compared with constants [5]. $R$ is the set of relations between node vertices $R \subset\left(C_{l} \times C_{r}\right)$ or $R: C_{l} \rightarrow C_{r}$.

The parameter $S$ (a consequence of the rule) is determined similarly to the parameter $C$. 


$$
S=<S_{l}, R, S_{r}>
$$

where $S_{l}$ is the left node consequence of the rule, $R$ is the relationship between the nodes of the rule, $S_{r}$ is the right node consequence of the rule.

$$
\begin{gathered}
S_{l}=F S_{l}\|\operatorname{Null}\| S, \\
S_{r}=F S_{r}\|\operatorname{Null}\| S,
\end{gathered}
$$

where $F S_{l}$ is the left final three of the consequence of the rule, $F S_{r}$ is the right final three of the consequence of the rule. Formulas (14) and (15) allow to describe the consequences with different degrees of nesting.

$$
\begin{aligned}
& F S_{l}=<L, \mathrm{Op}, W>, \\
& F S_{r}=<L, \mathrm{Op}, W>,
\end{aligned}
$$

where $L$ is the linguistic variable, Op is the operation, Op $=\{:=\}, W$ is the value of the consequence.

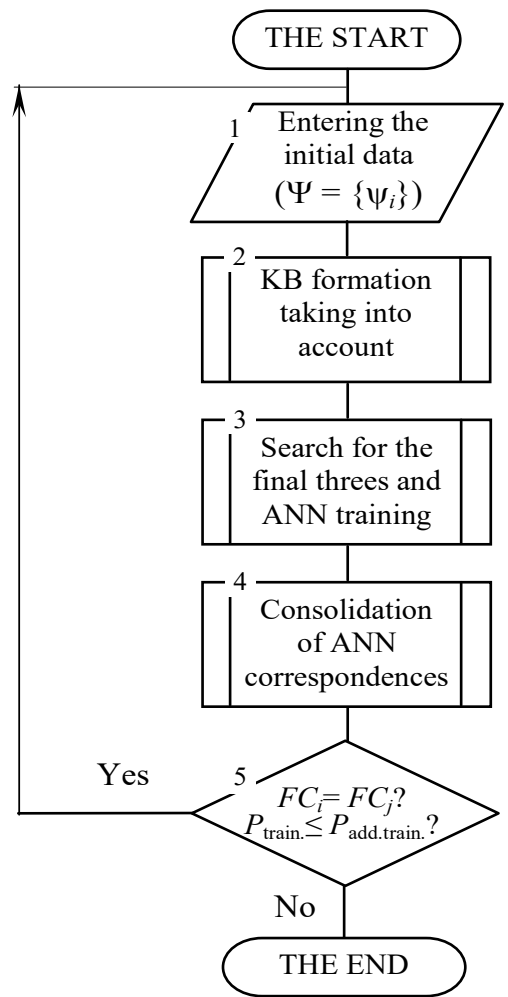

Fig. 1. The algorithm for implementing the proposed method

Step 3. Search for the finite threes and ANN learn (step 3 on the algorithm).

At this stage of the Rete method, the search for close finite triplets in all the rules of the production knowledge base is performed. The matches that were found between the final threes are denoted. The rules set out the references of such finite threes to ensure their one-time processing. In contrast to the classical neuro-fuzzy expert systems, in this neuro-fuzzy expert system as an artificial neural network it is proposed to use a neuro-fuzzy evolutionary network, the architecture of which is given in the work $[3,10]$. Also, here is the training of parameters 
and architecture of the artificial neural network in accordance with the method of training that is proposed in the work [3].

Let's consider the algorithm for finding the correspondences of the finite triples of the decision tree.

Input data: Rule is a database of rules that is presented in the form of a decision tree.

Output data: Rule' is an abbreviated database of rules that is represented as a decision tree. Intermediate data: $F C_{i}$ and $F C_{j}$ are the current final threes.

Step 3. 1. Initially, the work of the all the final three were not noticed (not checked), $m$ is the number of final threes. Find out the initial value $i=1$.

Step 3. 2. If $i>m$, then move to step 3. 10.

Step 3. 3. If $F C_{i}$ was mentioned, then $i=i+1$ and move to step 3. 2.

Step 3. 4. Choose $F C_{i}$. Find out $j=j+1$.

Step 3. 5. If $j>m$, then mark $F C_{i}$ as revised final three and move to step 3. 2.

Step 3. 6. If $F C_{j}$ was mentioned, then $j=j+1$ and move to step 3. 5.

Step 3. 7. Choose $F C_{j}$. Perform the procedure of checking the proximity of the end nodes and end triplets $F C_{i}$ and $F C_{j}$.

Step 3. 8. If the result is successful, then add $F C_{j}$ in the list of matches for $F C_{i}, F C_{j}$ and the final three that was tested.

Step 3. 9. Determination of a learning error. Decision-making on the training of ANN taking into account the type of uncertainty.

Step 3. 10. Move to step 3. 2.

Step 3. 11. The end. algorithm).

Step 4. Consolidation of correspondences and training of ANN (action 4 on the scheme of

At this stage, a recursive procedure is performed to check the proximity of intermediate nodes of decision trees. This procedure provides aggregation of correspondences between conditions in the rules of the knowledge base. Also at this stage is the training of architecture and parameters of ANN.

Then, let's consider the algorithm for finding the aggregation of the found matches.

Input data: Rule' is an abbreviated rule base, presented as a decision tree, with the same end triplets combined.

$S_{p}$ is the list of final triplets for which matches are found; $k$ is the number of items in the list $S_{p} . S_{p i}$ is the list of the final three $F C_{i}$, containing the corresponding end triplets with indices; $k_{i}$ is the number of items in the list $S_{p i}$.

Output data: Rule" - an abbreviated database of rules, which combines all the same conditions.

Intermediate data: $F C_{i}$ and $F C_{j}$ are the current final threes, $C_{i}$ and $C_{j}$ are the parent nodes for $F C_{i}$ and $F C_{j}$.

Step 4. 1. Find out $i=1$.

Step 4. 2. Choose in the decision tree $F C_{i}$, what is on the $i$-th place in the list $S_{p}$.

Step 4. 3. Find out $j=1$.

Step 4. 4. Select from the list $S_{p i}$ of the final three $F C_{j}$, which is in the $j$-th place. Delete parent nodes $C_{i}$ and $C_{j}$ for $F C_{i}$ and $F C_{j}$.

Step 4. 5. Make a recursive procedure to check intermediate nodes $C_{i}$ and $C_{j}$.

Step 4. 6. If the result of the function is successful, match the nodes $C_{i}$ and $C_{j}$, otherwise it is necessary to go to step 4.7.

Step 4. 7. $j=j+1$. If $j>k_{i}$, then to the step 4. 8, otherwise to the step 4. 4 .

Step 4. 8. $i=i+1$. If $i>k$, then to step 4. 10, otherwise to step 4. 2 .

Step 4. 9. The determination of a learning error. Decision-making on the training of ANN taking into account the type of uncertainty.

Step 4. 10. The end.

Step 5. The check of the metrics for assessing the proximity and determining the error of ANN learning (step 5 on Fig. 1). 
At this stage, the metrics of proximity of the obtained decisions are determined and the learning error is determined in order to make management decisions.

\section{Discussion of results on development of the improved method of search of the decision}

Simulation of the proposed method was performed in the software environment MathCad 2014.

To evaluate the effectiveness of the proposed method, modeling was performed using the following components:

- personal computer with installed special software and MathCad 2014;

- Agilent OmniBER 718 digital flow analyzer with software and a set of connecting cables that measures parameters;

- interference detector TRC $274 \mathrm{H} / \mathrm{V} / \mathrm{UHF}$ Jammer (20-3000 MHz), which simulated the operation of the electronic warfare system (transmitter power - $20 \mathrm{~W}$. Suppressive bandwidth is $10 \mathrm{MHz}$; type of interference is the noise interference with frequency manipulation, strategy of the EW-dynamic complex);

- MikroTik NetMetal 5 broadband radio access stations with the following parameters (128 positional quadrature amplitude manipulation; radiation bandwidth $40 \mathrm{MHz}$, radiation power $1 \mathrm{~W}$; radiation frequency $2.1-3 \mathrm{GHz}$ ).

The following linguistic variables were used to solve this problem:

1. Bit error probability (BER): The range of valid values: $10^{-3} \div 10^{-12}$;

$\mathrm{BER}=$ "Bit error probability" $=\{$ "channel is unusable", "limited channel", "channel is operational";;

2. Channel overlap factor: Range of allowable values: $0 \div 1$;

$\mathrm{KOV}=$ "Channel overlap factor"= $\{$ “channel completely blocked by interference", "channel partially blocked by interference", "channel without interference"\};

3. Interference transmitter power: Range of permissible values: $1 \div 20 \mathrm{~W}$;

$\mathrm{PJ}=$ "Interference transmitter power"=\{"low power", "medium power", "high power" $\}$;

4. Frequency of radiation of the transmitter of interference: Range of admissible values: $20 \div 3000 \mathrm{MHz}$;

$\mathrm{FJ}=$ "Frequency of interference transmitter radiation"=\{"Lower frequency band", "middle frequency band", "upper frequency band"\};

5. Power of broadband radio access station: Range of admissible values: $0 \div 1 \mathrm{~W}$;

$\mathrm{PW}=$ "Broadband Radio Station Power"=\{"low power", "medium power", "high power" $\}$;

6. Type of signal-code design of broadband radio access station: Range of admissible values: $4 \div 128$ positional quadrature amplitude manipulation;

$\mathrm{SC}=$ "Type of signal-code design of broadband radio access station"=\{"low position", "medium position", "high position"\};

7. Frequency of radiation of the broadband radio access station: Range of admissible values: $2100 \div 3000 \mathrm{MHz}$;

$\mathrm{FW}=$ "Broadband radio station frequency"=\{“Lower band", "middle band", "upper band" $\}$;

8. Uncertainty of the electronic environment: Range of acceptable values: complete uncertainty $\div$ complete knowledge;

$\mathrm{UN}=$ "Uncertainty of the electronic environment" $=\{$ “Complete uncertainty", "partial uncertainty", "complete knowledge"\};

To simplify further writing, denote the fuzzy variables "zero" - "Z", "low" - "L", "normal" "N", "high" - "H".

The expert (operator of the broadband radio access station) performed the initial adjustment of the membership functions of the terms of the set of neuron-fuzzy expert system, because all sources of radio radiation have different characteristics. The expert indicated which values of the primary and calculated parameters should be considered high for a given broadband radio access station, which are medium and which are low. The membership functions for the analysis of the electronic environment are presented in the specified form according to the formula 


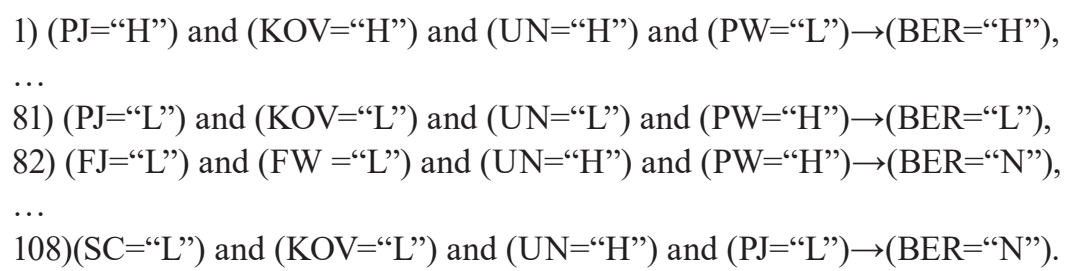

This example provides part of the neural fuzzy expert system rule base. In the basic base of rules there are rules not only with connections of conditions by devices of T-norms, but also by devices of T-conorms and with negations of conditions.

In the worst case, to find a solution, the system should check all the rules contained in the rules database. That is, it is necessary to check 405 conditions and calculate 297 operations of the T-norm. This is an unacceptably long process, given the limitations of the hardware.

The input data for the neuro-fuzzy expert system are the transmitter power of the broadband access station, the type of signal-code design of the broadband radio access station, the uncertainty of the REE (the share of parameters known about the interference detector), the frequency of the broadband radio access station. After passing the fuzzification stage, the system received fuzzy estimates for each controlled parameter.

For example, if the bit error probability value in the channel is $\mathrm{BER}=10^{-3}$, the power of the broadband radio access station is maximum and is $1 \mathrm{~W}$, 4-position quadrature amplitude manipulation is used, REE uncertainty is full, the frequency of the broadband radio access station is lower and these values for a given station, as "low", the rule from the knowledge base will be executed, as a result of which the following conclusion will be obtained: the jammer is high, the channel spectrum overlap is full, the jammer and the broadband access station operate at the same frequency. Therefore, it is necessary to adjust the radiation frequency of the broadband radio access station="high". Then the value of "high" of the linguistic variable "radiation frequency of the broadband radio access station" was defuzzified, and a new value of the radiation frequency to be set was transmitted to the broadband radio access station.

Let's evaluate the complexity $\Xi_{\text {mod Rete }}(n, m, k, t, s)$ of finding a solution for a neuro-fuzzy expert system operating on the basis of a modified Rete method. It is necessary to first form an estimate of the complexity of the neuro-fuzzy expert system with the classical method of finding a solution $\Xi_{\text {Rete }}(n, m)$ and then estimate the reduction in the number of calculations.

Let it be that the $n$-number of rules in the neuro-fuzzy expert system, $m_{i}$ - the number of conditions in the $i$-th rule $(i=1, . ., n), k$ is the number of different linguistic variables involved in the rules, $t_{i}$ is the the power of the term set of the $i$-th linguistic variable involved in the conditions rules, $s$ is the number of relationships between variables in the conditions.

It should be noted that the modified Rete method reduces the number of iterations while searching for a solution and reduces the number of calculations on each iteration.

In this case, the average complexity of performing one cycle of checking the rules in the operation of a neuro-fuzzy expert system with the classical method will look like (18):

$$
\begin{gathered}
\Xi_{\text {Rete }}(n, m)=\sum_{i=1}^{n}\left(2 \times m_{i}-1\right), \\
\Xi_{\text {mod Rete }}(n, m, k, t, s)=\sum_{i=1}^{n}\left(m_{i}-1\right)+\min \left\{\sum_{i=1}^{n}\left(m_{i}\right), \sum_{i=1}^{k}\left(t_{i}\right) \times s\right\},
\end{gathered}
$$

where the complexity of processing of t-norms or t-conorms is calculated, and then the minimum value from which complexity of all conditions of rules and complexity of all combinations of elements of term sets of variables and signs of relations is calculated.

Estimate the complexity for the rule base (RB) is given in Table 1. 
To compare the efficiency of the assessment, the classical method Rete, Treat and Leaps and the proposed method were used [10-12].

This table clearly shows that the use of the modified Rete method is justified for database rule that contain a large number of rules and a relatively small number of linguistic variables. In this case, the modified Rete method allows to speed up information processing almost twice in comparison with the fuzzy expert system, and by $20-25 \%$ in comparison with the classical Rete method.

Table 1

The value of the complexity estimation

\begin{tabular}{ccccccccccc}
\hline & $\boldsymbol{n}$ & $\boldsymbol{m}_{c e p}$ & $\boldsymbol{k}$ & $\boldsymbol{t}_{\text {cep }}$ & $\boldsymbol{s}$ & $\boldsymbol{\Xi}_{\text {HEC }}$ & $\boldsymbol{\Xi}_{\text {Rete }}$ & $\boldsymbol{\Xi}_{\text {Treat }}$ & $\boldsymbol{\Xi}_{\text {Leaps }}$ & $\boldsymbol{\Xi}_{\text {modRete }}$ \\
\hline RB 1 & 20 & 9 & 12 & 5 & 6 & 150 & $\mathbf{1 5 0}$ & 150 & 150 & $\mathbf{1 5 0}$ \\
RB 2 & 200 & 9 & 12 & 5 & 6 & 1500 & $\mathbf{1 4 2 0}$ & 1550 & 1580 & $\mathbf{1 1 4 0}$ \\
RB 3 & 400 & 9 & 12 & 5 & 6 & 3450 & $\mathbf{3 3 0 0}$ & 3500 & 3550 & $\mathbf{2 6 6 0}$ \\
RB 4 & 600 & 9 & 12 & 5 & 6 & 7000 & $\mathbf{6 5 6 0}$ & 6600 & 6690 & $\mathbf{5 0 5 0}$ \\
RB 5 & 20 & 9 & 12 & 5 & 6 & 150 & $\mathbf{1 5 0}$ & 150 & 150 & $\mathbf{1 5 0}$ \\
RB 6 & 200 & 9 & 12 & 5 & 6 & 1500 & $\mathbf{1 4 2 0}$ & 1550 & 1580 & $\mathbf{1 1 4 0}$ \\
RB 7 & 400 & 9 & 12 & 5 & 6 & 3450 & $\mathbf{3 3 0 0}$ & 3500 & 3550 & $\mathbf{2 6 6 0}$ \\
RB 8 & 600 & 9 & 12 & 5 & 6 & 7000 & $\mathbf{6 5 6 0}$ & 6600 & 6690 & $\mathbf{5 0 5 0}$ \\
RB 9 & 20 & 9 & 12 & 5 & 6 & 150 & $\mathbf{1 5 0}$ & 150 & 150 & $\mathbf{1 5 0}$ \\
RB 10 & 200 & 9 & 12 & 5 & 6 & 1500 & $\mathbf{1 4 2 0}$ & 1550 & 1580 & $\mathbf{1 1 4 0}$ \\
RB 11 & 400 & 9 & 12 & 5 & 6 & 3450 & $\mathbf{3 3 0 0}$ & 3500 & 3550 & $\mathbf{2 6 6 0}$ \\
RB 12 & 600 & 9 & 12 & 5 & 6 & 7000 & $\mathbf{6 5 6 0}$ & 6600 & 6690 & $\mathbf{5 0 5 0}$ \\
RB 13 & 20 & 9 & 12 & 5 & 6 & 150 & $\mathbf{1 5 0}$ & 150 & 150 & $\mathbf{1 5 0}$ \\
RB 14 & 200 & 9 & 12 & 5 & 6 & 1500 & $\mathbf{1 4 2 0}$ & 1550 & 1580 & $\mathbf{1 1 4 0}$ \\
RB 15 & 400 & 9 & 12 & 5 & 6 & 3450 & $\mathbf{3 3 0 0}$ & 3500 & 3550 & $\mathbf{2 6 6 0}$ \\
RB 16 & 600 & 9 & 12 & 5 & 6 & 7000 & $\mathbf{6 5 6 0}$ & 6600 & 6690 & $\mathbf{5 0 5 0}$
\end{tabular}

The results of the assessment of the state of the electronic environment for the different systems are presented in Fig. 2.

Features of systems of the analysis of a radio electronic situation are: a large number of the analyzed parameters; dynamic change of the electronic environment; functioning in conditions of uncertainty about the state of the electronic environment; constant updating of the signal base; functioning in the conditions of influence of natural and intentional disturbances. At the same time, in the conditions of constant replenishment of knowledge bases, the use of the classical Rete method is unacceptable from the point of view of adequacy and correctness of calculations.

The research of the developed method showed that this method provides an average of 20-25\% higher efficiency of assessment and does not accumulate errors during training (Table 1).

The main advantages of the proposed evaluation method are:

- it does not accumulate learning errors during the search for solutions by learning artificial neural networks (adjusting the parameters and architecture of the artificial neural network);

- less computational complexity, Table 1 (20-25\% increase in the efficiency of decision-making);

- while performing a search for a solution, the same conditions are calculated once, which provides acceleration of the cycle of revision of the rules;

- less memory, as references to them are used instead of the same terms of the rules;

- it allows to define not only separate identical conditions in base of rules, but also blocks of identical conditions that allows to increase speed of search of the decision in addition;

- it allows to work with clear and fuzzy products;

- taking into account the type of uncertainty about the state of the electronic environment. 


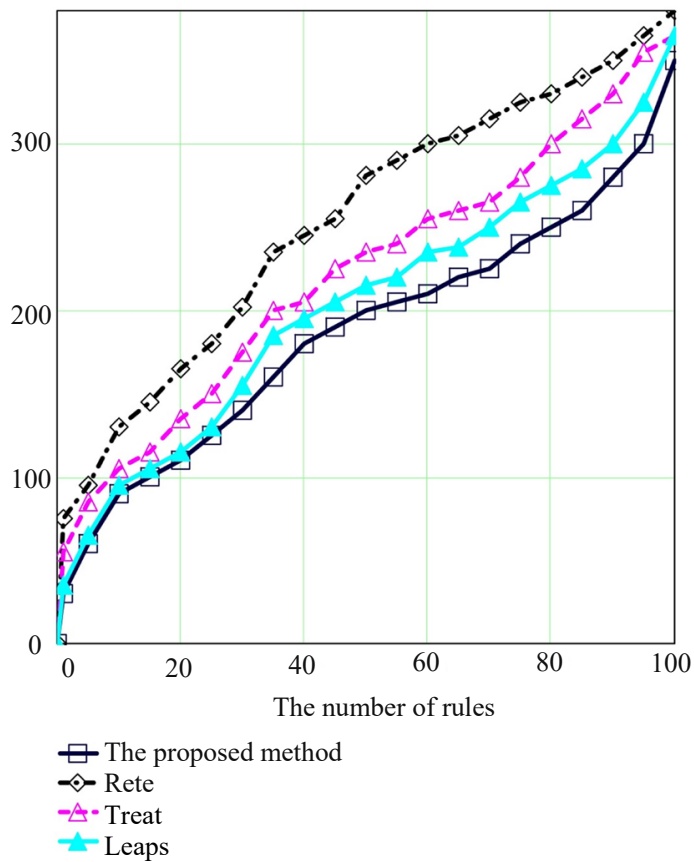

Fig. 2. The comparison of the efficiency of the obtained assessment for different methods

As for the limitations of this method, it is adapted for the analysis of the electronic environment, in terms of its uncertainty and high dynamics. However, the proposed method is able to successfully solve the problem of data analysis with appropriate adaptation to a particular type of decision support systems for REE analysis.

However, as already mentioned, in the course of the known methods accumulate errors, which is why this method proposes the use of artificial neural networks that are evolving. The results of the evaluation of efficiency are shown in Fig. 3.

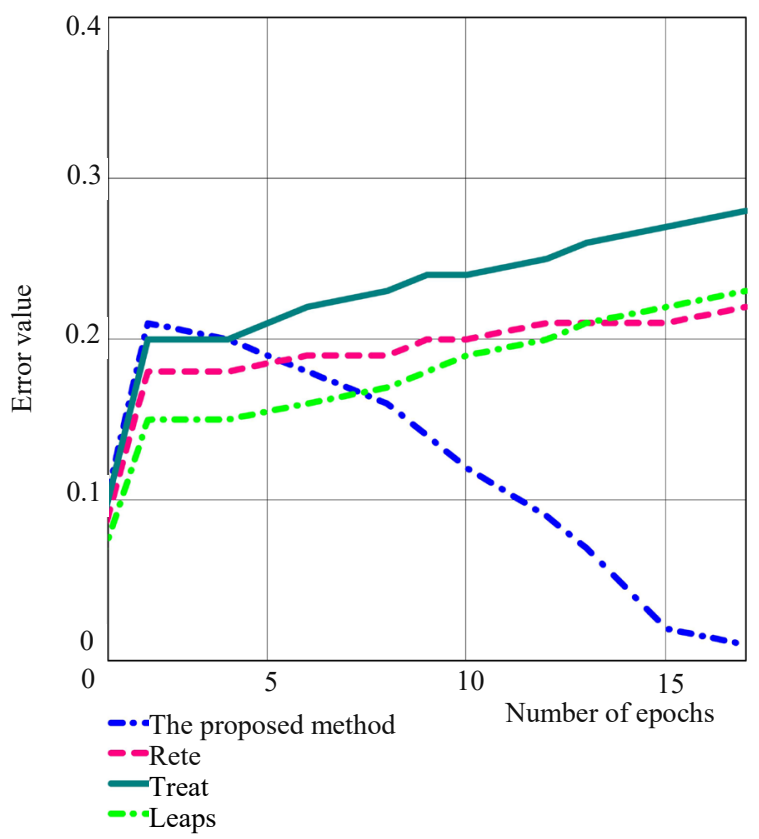

Fig. 3. The evaluation of the effectiveness of the use of evolving artificial neural networks

Fig. 3 shows that the use of artificial neural networks, which evolves, allows after 3 epochs not to accumulate learning errors and there is a gradual reduction of learning errors. 
Consider the cost of learning an evolving artificial neural network.

A training sample containing data on the radio source was used for the simulation. The rule base from this sample was used for modeling.

The proposed method, Rete, Treat and Leaps were used to compare the costs of ANN training.

The results of the comparison for different methods are presented in Table 2.

Table 2

The comparison of the results for different methods

\begin{tabular}{ccccc}
\hline Name of the system & $\begin{array}{c}\text { Number of } \\
\text { the parameters }\end{array}$ & $\begin{array}{c}\text { The number of conditions } \\
\text { of the calculated rules }\end{array}$ & $\begin{array}{c}\text { The number of rules } \\
\text { in the database }\end{array}$ & Time, sec. \\
\hline Rete & 8 & 350 & 100 & 0.1398 \\
Treat & 8 & 350 & 100 & 0.1501 \\
Leaps & 8 & 350 & 100 & 0.1456 \\
Proposed evolving & 8 & 387 & 100 & 0.954 \\
ANN method & & &
\end{tabular}

As it can be seen from Table 2, for the training of ANN, it is necessary to calculate an additional 37 rules (training sample, $10.5 \%$ ), which increases the computational complexity for an artificial neural network learning, but gives a gain in efficiency at 20-25\% in comparable to the classical Rete method.

The disadvantages of the proposed method are:

- the loss of the informativeness in the assessment due to the construction of the membership function. This loss of information can be reduced by choosing the type of membership function and its parameters in the practical implementation of the proposed method in support and decision-making systems. The choice of the type of the membership function depends on the computing resources of a particular electronic computing device;

- lower accuracy of assessment on a single parameter of condition assessment;

- loss of accuracy of results during the reconstruction of the architecture of the artificial neural network.

This research is a further development of research that was conducted by the authors, aimed at developing the theoretical foundations for improving the efficiency of artificial intelligence systems and published earlier [1-3].

Areas of further research should be aimed at reducing the computational costs in the processing of various types of data in special purpose systems.

\section{Conclusions}

An improved method for finding solutions for neuro-fuzzy expert systems for analyzing the electronic environment has been developed.

The differences between the proposed method from the known, which determines its novelty, are as follows:

- while assessing the electronic situation, the type of uncertainty is additionally taken into account in accordance with expressions (10)-(12) [2];

- evolving artificial neural networks were used to increase the efficiency of information processing [2];

- ability to work with both clear and fuzzy products through the use of evolving artificial neural networks;

- no accumulation of the training error of artificial neural networks as a result of processing of the information arriving on an input of artificial neural networks at the expense of training of architecture and parameters.

The use of the proposed method was tested on the example of assessing the state of the electronic environment. This example showed an increase in the efficiency of evaluation at the level of $20-25 \%$ on the efficiency of information processing. 


\section{References}

[1] Kuchuk, N., Mohammed, A. S., Shyshatskyi, A., Nalapko, O. (2019). The method of improving the efficiency of routes selection in networks of connection with the possibility of self-organization. International Journal of Advanced Trends in Computer Science and Engineering, 8 (1). 1-6. Available at: http://www.warse.org/IJATCSE/static/pdf/file/ijatcse01812sl2019.pdf

[2] Sova, O., Turinskyi, O., Shyshatskyi, A., Dudnyk, V., Zhyvotovskyi, R., Prokopenko, Y. et. al. (2020). Development of an algorithm to train artificial neural networks for intelligent decision support systems. Eastern-European Journal of Enterprise Technologies, 1 (9 (103)), 46-55. doi: https://doi.org/10.15587/1729-4061.2020.192711

[3] Mikhaylov, R. L., Makarenko, S. I. (2013). Estimating Communication Network Stability Under the Conditions of Destabilizing Factors Affecting it. Radiotehnicheskie i telekommunikatsionnye sistemy, 4, 69-79.

[4] Bodyanskiy, E. V., Strukov, V. M., Uzlov, D. J. (2017). Generalized metrics in the problem of analysis of multidimensional data with different scales. Zbirnyk naukovykh prats Kharkivskoho universytetu Povitrianykh Syl, 3, 98-101.

[5] Semenov, V. V., Lebedev, I. S. (2019). Processing of signal information in problems of monitoring information security of unmanned autonomous objects. Scientific and Technical Journal of Information Technologies, Mechanics and Optics, 19 (3), 492-498. doi: https://doi.org/10.17586/2226-1494-2019-19-3-492-498

[6] Zhou, S., Yin, Z., Wu, Z., Chen, Y., Zhao, N., Yang, Z. (2019). A robust modulation classification method using convolutional neural networks. EURASIP Journal on Advances in Signal Processing, 2019 (1). doi: https://doi.org/10.1186/s13634-019-0616-6

[7] Shaheen, E. M., Samir, M. (2013). Jamming Impact on the Performance of MIMO Space Time Block Coding Systems over Multi-path Fading Channel. REV Journal on Electronics and Communications, 3 (1-2). doi: https://doi.org/10.21553/rev-jec.56

[8] Malik, S., Kumar, S. (2017). Optimized Phase Noise Compensation Technique using Neural Network. Indian Journal of Science and Technology, 10 (5), 1-6. doi: https://doi.org/10.17485/ijst/2017/v10i5/104348

[9] Rotshteyn, A. P. (1999). Intellektual'nye tehnologii identifikatsii: nechetkie mnozhestva, neyronnye seti, geneticheskie algoritmy. Vinnitsa: Universum-Vinnytsia, 295.

[10] Mazhara, O. A. (2015). Treat algorithm implementation by the basic match algorithm based on CLIPS programming environment. Elektronnoe modelirovanie, 37 (5), 61-75.

[11] Bolotova, S. Yu., Makhortov, S. D. (2011). Relevant backward inference algorithms based on solving the production logical equations. Iskusstvenniy intellekt i prinyatie resheniy, 2, 40-50.

[12] Zhyvotovskyi, R., Shyshatskyi, A., Petruk, S. (2017). Structural-semantic model of communication channel. 2017 4th International Scientific-Practical Conference Problems of Infocommunications. Science and Technology (PIC S\&T). doi: https:// doi.org/10.1109/infocommst.2017.8246454 